\title{
Le rôle économique et social des chemins de fer secondaires en Italie
}

\section{Stefano Maggi}

Traducteur : Patricia Vannetti

\section{OpenEdition \\ Journals}

Édition électronique

URL : https://journals.openedition.org/rhcf/2086

DOI : $10.4000 /$ rhcf.2086

Éditeur

Rails \& histoire

Édition imprimée

Date de publication : 2 mai 2002

Pagination : 370-394

ISBN : 00996-9403

ISSN : 0996-9403

Référence électronique

Stefano Maggi, «Le rôle économique et social des chemins de fer secondaires en Italie », Revue d'histoire des chemins de fer [En ligne], 24-25 | 2002, mis en ligne le 28 avril 2015, consulté le 22 avril 2022. URL : http://journals.openedition.org/rhcf/2086 ; DOI : https://doi.org/10.4000/rhcf.2086 


\section{Cinquième partie}

Comparaisons et perspectives 


\section{Stefano MAGGI}

(article traduit par Patricia Vannetti)

\section{Le rôle économique et social des chemins de fer secondaires en Italie}

\section{Introduction}

Quand on parle des voies ferrées secondaires en Italie, il faut d'abord remarquer qu'il n'existe aucune définition juridique qui les distingue des lignes principales, même si, au cours des premières décennies du XX siècle, le terme « secondaire » a fini par indiquer les chemins de fer dits : « donnés en concession », c'est-à-dire des chemins de fer gérés par des compagnies locales détachées du réseau national. Il faut aussi relever que même le réseau national, créé en 1905, a toujours comporté quelques sections présentant les traits caractéristiques des chemins de fer secondaires, tels qu'un tracé établi en vue de desservir les campagnes et les centres moins importants, un trafic limité, la fonction d'évacuation de produits essentiellement agricoles et, quelquefois, une clientèle touristique.

Quand on procéda à la nationalisation de 1905, le réseau national des chemins de fer comptait environ $17000 \mathrm{~km}$. Mais toutes les lignes n'étaient pas gérées par la compagnie nationale : en effet, environ $3000 \mathrm{~km}$ de voies ferrées et autant de lignes de tramways appartenaient à des entreprises privées ou municipales qui faisaient circuler un grand nombre de petits trains et de trams à vapeur ainsi que des tramways électriques.

Mais nous allons remonter aux débuts, aux années 1860-1870 pour reconstruire les rôles économique et social que les chemins de fer secondaires ont assumés en Italie.

\section{Les chemins de fer secondaires}

En Italie, l'essor des chemins de fer secondaires se place dans les vingt dernières années du XIX ${ }^{e}$ siècle ${ }^{1}$. Il fut caractérisé par un véritable mouvement général de promotion de la ramification du réseau ferroviaire accompagné par la publication d'une foule de brochures - conservées de nos jours dans les bibliothèques et archives locales - 
destinées à soutenir les projets de chaque ligne de périphérie. Ces brochures se ressemblaient toutes : dans leur introduction le spécialiste ou comité promoteur d'une ligne, auteur du texte, mettait en avant, quelquefois fort élégamment, la civilisation apportée par le train.

La diffusion de cette presse d'actualité mineure, dont les origines sont clairement positivistes, et qui représentait le train comme une source de progrès économique et social, fut très importante. Cette vision du train ne s'adressait pas seulement aux techniciens, aux économistes ou aux milieux cultivés mais aussi à la population tout entière, même et surtout celle des centres urbains de la périphérie du pays desservis par les voies ferrées secondaires. Tout le monde était donc concerné et s'intéressait à l'arrivée de la locomotive haletante, comme en témoignaient les réunions des comités promoteurs et, surtout, les cérémonies d'inauguration, toujours fréquentées par une foule de citoyens en liesse, rapportées ici et là par les chroniques.

À cette époque, on avait l'impression que le progrès s'étendait des villes jusqu'aux centres moins importants par le biais de trains plus petits que les trains habituels et par les trams; une image positive des rails se diffusait dans les campagnes, grâce à la ramification du réseau, et faisait sortir toujours plus de populations et de territoires de la léthargie du passé en les introduisant dans le marché, dans l'économie et dans la civilisation du XIX ${ }^{\mathrm{e}}$ siècle.

En effet, une fois les lignes principales terminées, les centres périphériques commencèrent eux aussi à réclamer à grands cris le train qui, dans une société agricole et dépourvue d'automobiles, semblait le seul lien avec la civilisation, capable d'apporter le développement économique et la modernisation politique et sociale. L'Italie se caractérisait par un réseau urbain antique constitué par un grand nombre de petites villes dynamiques, souvent situées sur les hauteurs pour échapper au paludisme, maladie endémique et séculaire : ce sont elles qui furent les protagonistes de la réalisation d'un réseau secondaire dans la péninsule grâce à leur activité politique municipale. Comme l'écrivait le journaliste et député Pacifico Valussi, observateur attentif de la société italienne :

«les villes secondaires, autrefois florissantes, se plaignent sans arrêt des effets néfastes que les voies ferrées provoquent chez elles, même si en fait elles les frôlent simplement ; mais même en les frôlant, elles emportent une partie de la vie locale dont ces centres jouissaient pour la déverser dans les grandes villes où les chemins de fer s'entremêlent $t^{2}$.» 
Autre facteur, dans les vingt dernières années du XIX ${ }^{\mathrm{e}}$ siècle, les progrès techniques des lignes à voie étroite qui permettaient des tracés aux courbes plus serrées et aux pentes plus raides que la voie normale sans avoir à construire des ouvrages grandioses et le succès de certains systèmes d'adhérence, comme la crémaillère, permirent de relier par le rail les nombreuses petites villes situées sur les collines ou sur les flancs des montagnes. Les communes de dimension moyenne investirent ellesmêmes de plus en plus dans le secteur grâce aux prêts qu'elles pouvaient contracter auprès de la banque publique : la « Caisse de dépôts et de prêts ».

En 1879 on promulga la « loi Baccarini », du nom du ministre des Travaux publics du moment $t^{3}$. Cette loi, similaire au plan Freycinet en France ${ }^{4}$, tentait de classer les « chemins de fer complémentaires » reconnus nécessaires à l'achèvement du réseau de la péninsule en quatre catégories. La quatrième et dernière catégorie était constituée par les "chemins de fer secondaires", à savoir $1530 \mathrm{~km}$ de lignes que l'on pouvait construire à condition que les Provinces et les Communes intéressées en prouvent l'utilité, qu'elles s'engagent à participer aux frais et démontrent leur capacité de le faire 5 .

La majeure partie des tronçons des chemins de fer secondaires, réalisés entre la fin du $\mathrm{XIX}^{\mathrm{e}}$ siècle et le début du XX $\mathrm{XX}^{\mathrm{e}}$ siècle, a été donnée en concession à des entreprises locales et municipales et n'étaient pas gérée par les compagnies principales. Après les réformes administratives générales de 1865 et de $1885^{\circ}$, certains chemins de fer moins importants étaient restés à des petites sociétés privées qui géraient, à la date du 31 décembre 1887, $1326 \mathrm{~km}$ de réseau : $414 \mathrm{~km}$ étaient attribués à la Compagnie royale sarde, $189 \mathrm{~km}$ à la Compagnie Palerme-Marsala-Trapani en Sicile ; parmi les réseaux plus étendus, on comptait la Société vénitienne qui avait $134 \mathrm{~km}$ de lignes dont la ligne Vicenza-Schio, la ligne Vicenza-Treviso et la ligne Padoue-Bassano; $63 \mathrm{~km}$ étaient gérés par les Chemins de fer du Nord-Milan et allaient considérablement augmenter au cours du nouveau siècle ; $134 \mathrm{~km}$ enfin étaient gérés par le Chemin de fer des Appennins du Centre, Arezzo-Fossato di Vico. Au début du $\mathrm{XX}^{\mathrm{e}}$ siècle d'autres lignes vinrent s'ajouter à cette liste, surtout les voies de communication avec les centres de montagne des Alpes et des Appennins et, en 1905 déjà, au lendemain de la nationalisation, l'extension globale du réseau des chemins de fer en concession avait $3419 \mathrm{~km}$ dont 2061 étaient à voie normale et 1358 à voie étroite 7 . 
Même si en Italie le kilométrage des lignes touristiques de montagne ou de moyennes montagne ne pouvait pas être comparé à celui de la Suisse, il n'était pas négligeable, au contraire ! Ces infrastructures furent réalisées dans les trente premières années du $\mathrm{XX}^{\mathrm{c}}$ siècle, quand le train arriva à Campo Tures, Predazzo, Ortisei, Cortina d'Ampezzo et Agordo dans les Dolomites; à Renon, La Mendola, Malè et Riva del Garda entre Trento et Bolzano ; à Piazza Brembana et Clusone dans les vallées bergamasques; à Vallombrosa, Gubbio et Norcia dans les Appennins du centre ; ̀̀ Castrovillari, San Giovanni in Fiore, Mammola, Cinquefrondi et Sinopoli dans la Calabre de l'intérieur; à Palazzo Adriano et Piazza Armerina, juste pour citer deux localités touchées par le dense réseau des chemins de fer siciliens à voies étroites; enfin, à Mandas, Sorgono et Arbatax pour ce qui concerne la Sardaigne.

En outre, là où l'investissement considérable nécessaire à un chemin de fer n'était pas justifié, on posait des rails sur les routes extra-urbaines et on réalisait ainsi des lignes de tramways à chevaux ou plus souvent à vapeur dotés de petites locomotives et de matériel roulant léger.

\section{Les tramways}

Le premier tram à chevaux fut inauguré en 1872 à Turin, et les tronçons extra-urbains furent étendus en 1876 entre Milan et Monza (traction hippomobile), tandis que la diffusion des lignes de tramways à vapeur commença en 1878 avec la ligne Milan-Vaprio d'Adda via Gorgonzola et la ligne Cuneo-Borgo San Dalmazzo ; les trams mus par l'électricité commencèrent à circuler en 1890 d'abord à Rome puis entre Florence et Fiesole.

Comparée à ce qu'elle fut ailleurs en Europe, en Belgique et en Hollande en particulier, en Italie aussi la diffusion de ces derniers fut exceptionnelle.

A la fin de l'année 1879, après les premières inaugurations, 29 lignes ayant au total $515 \mathrm{~km}$ de longueur étaient en service dans la péninsule : sur 17 lignes, appelées « lignes de tramways à vapeur» on « utilisait déjà la locomotive »" L'année suivante le nombre des kilomètres avait augmenté et l'on en comptait 700, dont la moitié dans la province de Milan. En 1885 déjà, 384 voitures de tramways circulaient sur le réseau à vapeur le plus étendu parmi les pays du continent ${ }^{10}$. Au $1^{\text {er }}$ octobre 1888 le réseau des tramways à vapeur comptait $2262 \mathrm{~km}$ : les rails étaient posés sur une longueur de $139 \mathrm{~km}$ de routes nationales, sur $1572 \mathrm{~km}$ de routes provinciales et $269 \mathrm{~km}$ de routes municipales; en revanche, $281 \mathrm{~km}$ de rails étaient posés en site propre. Les réseaux les plus importants se trouvaient en Lombardie $(905 \mathrm{~km})$ et dans le 
Piémont $(774 \mathrm{~km})^{11}$. Au 31 décembre 1902, l'extension des lignes de tramways avaient atteint $3541 \mathrm{~km}$, et ils étaient gérés par 77 sociétés de transport où travaillaient 12484 employés; on comptait 264 lignes fonctionnant à vapeur dans la plupart des cas, plus exactement $3067 \mathrm{~km}$ à vapeur contre $705 \mathrm{~km}$ à électricité12. En $19092950 \mathrm{~km}$ de lignes à vapeur contre $1077 \mathrm{~km}$ de lignes électriques ${ }^{13}$.

De véritables réseaux de trams furent donc construits dans le nord de l'Italie ; en revanche, au centre et dans le Sud, quelques tronçons seulement furent mis en service dont les plus importants étaient situés autour de Rome, Naples et Messine. La Société des tramways interprovinciaux de la plaine du Pô, qui gérait quelques lignes aboutissant à Milan, y compris celle vers Vaprio, devint la seconde entreprise de tramways du continent. On ne peut donc vraiment pas dire que ce secteur n'ait pas été important dans la société italienne de cette époquelà, bien au contraire! Mais le fait que la presse se soit peu intéressée aux tramways, contrairement à la curiosité qu'elle avait pour les chemins de fer, a toujours amené les reconstructions historiographiques à les laisser de côté au point même de les faire oublier parfois carrément, excepté par les collectionneurs de cartes postales.

La vapeur était le mode de traction le plus utilisé par les lignes extra-urbaines. Dans les villes, en revanche, on utilisa très tôt la traction électrique car elle était moins polluante, sauf à Ancône, Bergame et Gênes où l'on expérimenta les trams à vapeur. En Italie, les tramways adoptèrent en général la voie normale des chemins de fer $(1,445 \mathrm{~m})$, tandis que les voies de $1,10 \mathrm{~m}, 0,95$ et $0,75 \mathrm{~m}$ étaient plus rares. Comme pour les premiers chemins de fer, une bonne partie des premières entreprises de trams fut financée par des capitaux britanniques ou belges, et le mot « tranvai » alternait avec le mot anglais correspondant « tramway $»$.

Étant donné les ressources limitées de la population italienne, des tarifs bas furent adoptés et de ce fait les recettes des compagnies diminuèrent. Ces compagnies étaient par ailleurs étouffées, du moins quand on compare aux autres états européens, par des mesures de sécurité excessives qui étaient imposées par voie réglementaire, et qui rendaient obligatoire l'emploi d'un bon nombre d'agents : à bord d'un tram à vapeur de la ligne Milan-Magenta, par exemple, on comptait le mécanicien, le chauffeur de locomotive, le chef de train et deux conducteurs, c'est-à-dire à peu près le même personnel qu'un train de long parcours ; qui plus est, il y avait un garde-barrière à chaque intersection avec une route ${ }^{14}$. Du reste, les trams devaient marcher lentement car ils circulaient sur les mêmes routes que les véhicules ordinaires. La vitesse autorisée variait, selon les tronçons, de 5 à $20 \mathrm{~km} / \mathrm{h}^{15}$. 

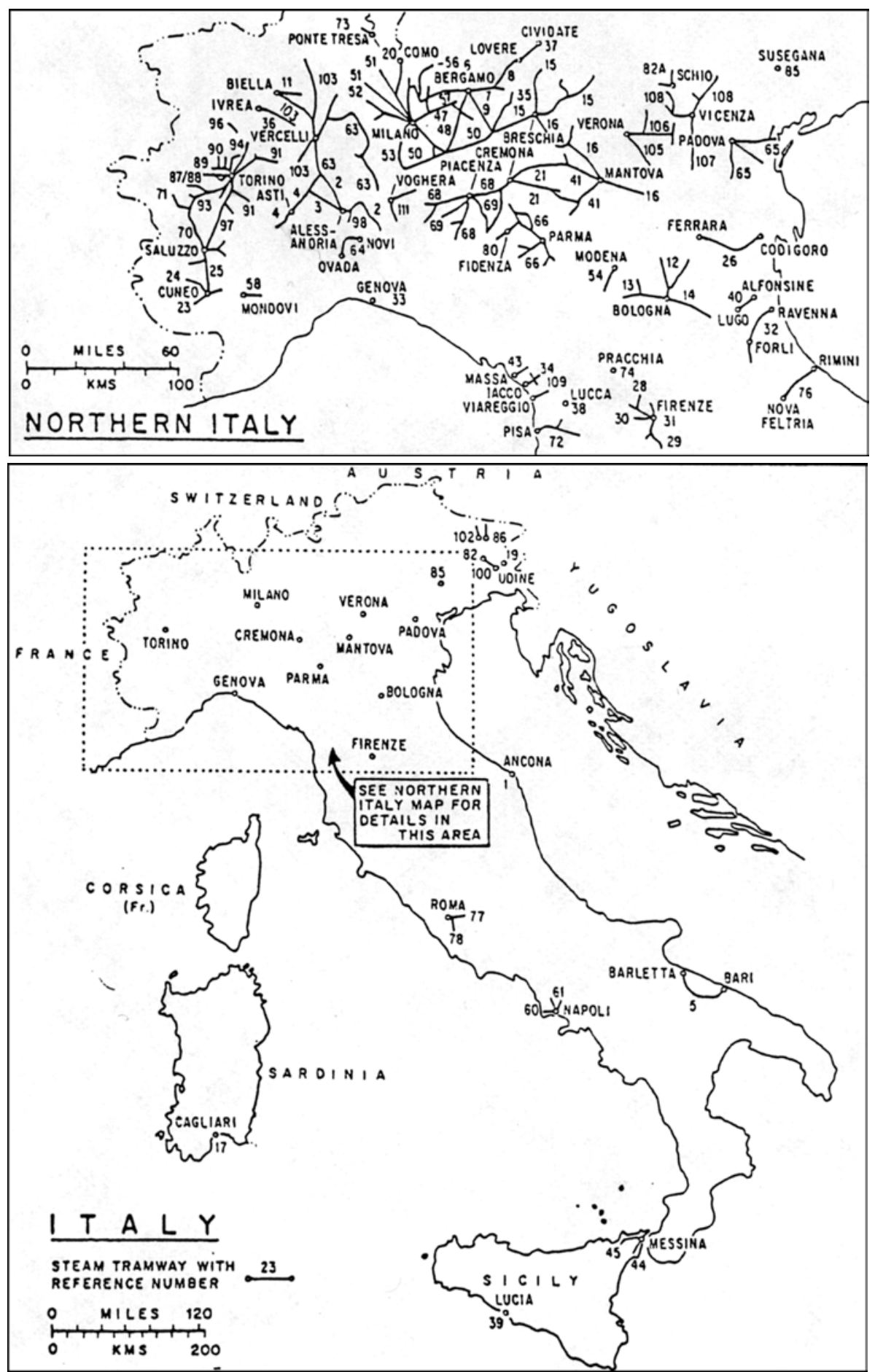

Source: G.E. Baddeley, The continental Steam Tram, Londres, 1980, p. 178

Figure 1 : Les réseaux de tramways en Italie. 


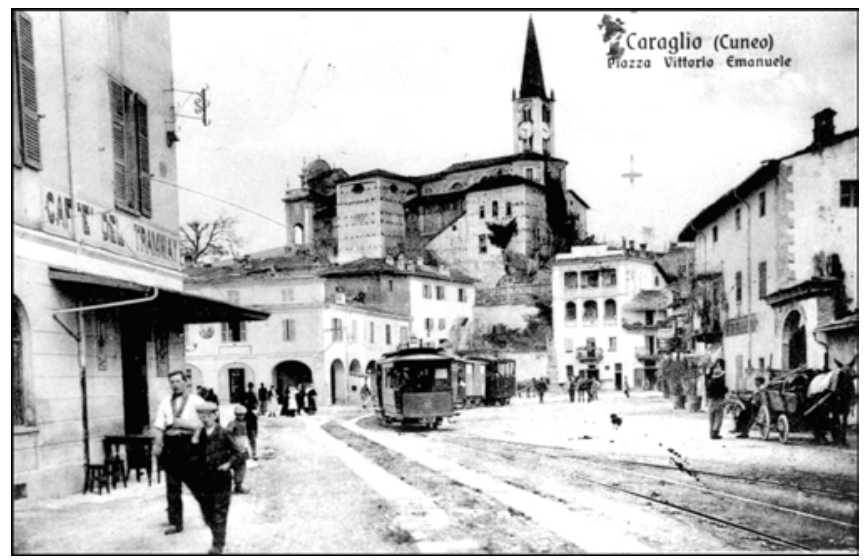

Tramway pour voyageurs et marchandises Cuneo - Dronero. A gauche on peut voir le "Cafè del tramway " (1910). Coll. Leonardo Carnesecchi, cl. d.r.

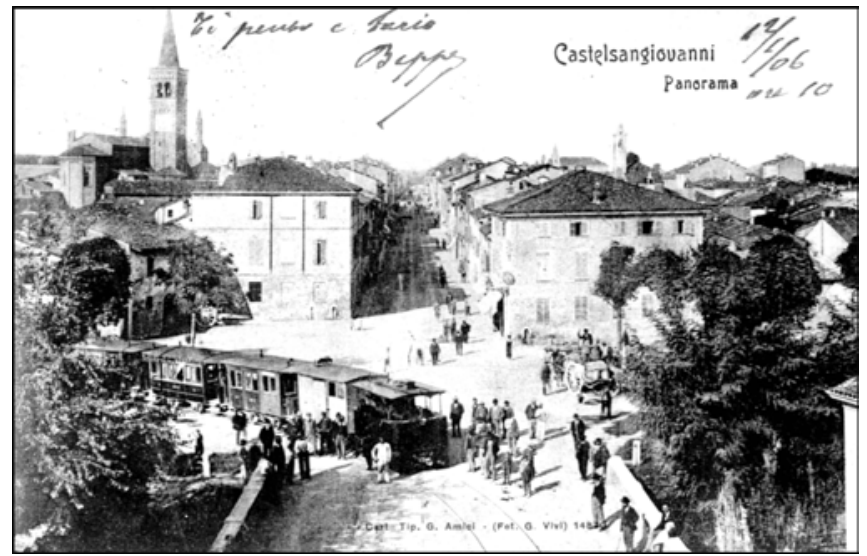

Tramway Castelsangiovanni - Pianello Val Tidone près de Piacenza (1906). Coll. Leonardo Carnesecchi, cl. d.r.

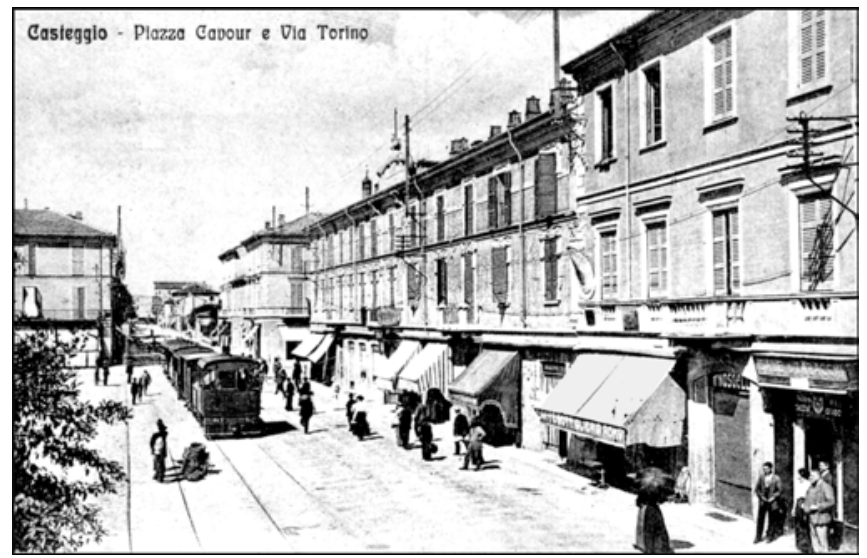

Tramway Voghera - Stradella (1920). Coll. Leonardo Carnesecchi, cl. d.r.

Figure 2 : Les tramways. 
De nombreuses lignes de tramways extra-urbains eurent un bon trafic de marchandises, constitué par des produits ruraux envoyés dans les villes ou transportés par le tram jusqu'aux gares de chemins de fer, dans les cas où il existait une intégration entre les deux moyens de transport. Le trafic était constitué aussi, réciproquement, par des produits manufacturés destinés aux centres de périphérie.

\section{Rôle économique et social}

Le rôle économique et social des tramways, tout comme celui des chemins de fer secondaires, peut être observé très facilement dans le secteur des marchandises, au vu des statistiques relatives au trafic de fret par ligne ou par gare : quels que soient les chiffres en effet on remarque partout une hausse sensible du trafic après les premières années qui suivirent l'inauguration, ce qui veut bien dire que l'Italie des périphéries avait rapidement changé à partir de la fin du XIX siècle, changement rendu possible en retour grâce à l'arrivée du rail.

Au début, il n'existait aucune différence juridique entre les lignes de tramways et les chemins de fer secondaires, appelés ordinairement « chemins de fer économiques » : la différence ressortait seulement sur le plan technique puisque les chemins de fer avaient leur site propre tandis que les tramways se trouvaient en majorité sur les routes. Cette lacune de la législation entraina d'importants problèmes pour leur construction et leur gestion : les chemins de fer économiques entraient davantage que les tramways, par analogie, dans les normes établies pour les chemins de fer ; les premiers tramways en revanche se développèrent en l'absence de règles qui auraient été prévues pour accompagner leur réalisation. Après maintes discussions, on décida - par un avis du Conseil d'État - que la concession du domaine routier pour y poser des rails revenait aux administrations propriétaires des routes, c'est-àdire aux Communes, à l'administration de la Province ou au ministère des Travaux publics pour les routes nationales; mais l'autorisation du ministère (approuvée par décret royal) était toujours nécessaire en cas de traction mécanique. Ces principes furent ensuite reconnus formellement par la première loi en la matière, promulguée en décembre 1896, après avoir trainé au parlement pendant une dixaine d'années ${ }^{16}$; cette loi, qui établit une distinction précise entre les chemins de fer et les tramways, redressa une situation confuse où l'on ressentait clairement que le progrès technique prenait désormais un train beaucoup plus rapide que la loi. Dans cette période, l'Italie se mettait au diapason de l'Europe et comblait les retards économique, social et technologique qui caractérisaient la société italienne au lendemain de l'unification nationale. 
Le rôle des chemins de fer secondaires et des tramways fut réellement fondamental dans certaines régions, surtout dans le nord de l'Italie où le réseau s'était développé le plus, grâce aussi à la meilleure situation financière des administrations locales et à une orographie plus simple, puisque les plaines y sont en majorité. Au centre-sud où prédominent collines et montagnes, l'extension des lignes fut moins importante, surtout celle des tramways, tandis que l'on construisit beaucoup de chemins de fer secondaires, en majorité à voie étroite ; on répondait ainsi à la demande insistante des populations locales qui considéraient le train comme le vecteur principal de la modernisation économique et sociale.

Les chemins de fer secondaires, financés surtout par l'État, se diffusèrent des zones les plus riches aux zones marginales au point de créer de véritables réseaux dans des régions pauvres comme la Basilicate, la Calabre, la Sicile et la Sardaigne. Les tramways en revanche, confiés aux capitaux privés ou municipaux, prospérèrent dans les régions les plus riches de la péninsule, celles qui avaient la plus grande densité de population et qui étaient caractérisées par un échange intense entre bourgs voisins et entre centres agricoles et villes correspondantes. C'est à Milan et à Turin que les lignes de tramways couvraient l'étendue la plus vaste : elles servirent de ce fait à transporter les premiers banlieusards, les jeunes provenant de la campagne et qui se rendaient tous les jours au travail dans les usines de ces deux grandes villes. Dans d'autres cas, comme par exemple celui de la ligne de tramway du Chianti - qui reliait Florence et Greve - et surtout celui de la ligne secondaire Sant'Ellero-Vallombrosa, toujours en Toscane ${ }^{17}$, les trams transportaient aussi des représentants de milieux plus aisés qui accompagnaient leur famille à la campagne et favorisaient ainsi le développement du tourisme dans sa forme la plus simple, c'est-à-dire la classique promenade champêtre.

Cependant, les lignes de tramways et les chemins de fer secondaires ont aussi servi à rapprocher le train des habitants. En Italie en effet, l'utilisation des chemins de fer fut longtemps limitée à cause des bas salaires et, aux alentours de 1910, la population prenait le train en moyenne deux fois par an par habitant contre 27 fois au RoyaumeUni. Les trains légers et les trams, avec leurs tarifs plus modérés, permirent en revanche d'élargir l'utilisation de ce transport mécanisé aux milieux ouvriers, qui se déplaçaient auparavant à pied ou ne se déplaçaient jamais.

Quand l'expansion des chemins de fer locaux atteignit son point culminant, on proposa à maintes reprises d'étendre le réseau en concession en confiant à de petites entreprises quelques lignes appartenant aux chemins de fer nationaux mais au trafic limité. En 1918, par exemple, Pietro Lanino, président du collège des ingénieurs des chemins de fer 
italiens, soutenait qu'il était nécessaire de « décharger la lourde structure des Chemins de fer de l'État en la débarrassant de la gestion de certains chemins de fer secondaires et locaux qui pourraient passer par contre à l'industrie privée et donc trouver une utilisation plus efficace $»^{18}$. Mais ces propositions ne furent jamais adoptées et l'on ne fit aucune réforme organisationnelle supplémentaire. C'est pourquoi en Italie la question des chemins de fer secondaires ne fut jamais résolue avec efficacité : certaines lignes furent conservées ou acquises par le réseau national, tandis que les autres, celles qu'on appelait « chemins de fer en concession ", furent gérées par des entreprises privées où le capital public augmentait parallèllement à la hausse du déficit du budget.

\section{La " taille des branches mortes "}

En Italie les tramways extra-urbains ont commençé dans les années 1930 déjà à suivre une courbe descendante, et les tronçons restants n'assuraient plus que le trafic des voyageurs. Leur élimination eut des raisons économiques et politiques : on pensait en effet que les rails posés sur les routes allaient gêner l'essor de l'automobile et qu'il fallait donc - comme l'affirmait le ministre des Communications Costanzo Ciano - «prendre son courage à deux mains et arracher les rails $»^{19}$. Le réseau des tramways extra-urbains, qui comptait $4217 \mathrm{~km}$ en 1930, ne mesurait plus que $2230 \mathrm{~km}$ en 1939. Pendant ces dix années le nombre des voyageurs transportés augmenta faiblement, passant de 179 à 192 millions tandis que les tonnes de marchandises diminuèrent, de 4300000 à $1900000^{20}$.

Le réseau des chemins de fer secondaires atteignit de son côté, dans le deuxième après-guerre, en 1955, son étendue la plus vaste avec $21923 \mathrm{~km}$; mais à partir de cette année-là, date du lancement commercial de la Fiat 600 - voiture qui marqua la motorisation de masse en Italie - on commença à supprimer ce qu'on appelait "les rameaux secs », les branches mortes. $2100 \mathrm{~km}$ de lignes furent graduellement mises hors de service, surtout parmi celles données en concession, car les Chemins de fer de l'État ne perdirent que $600 \mathrm{~km}$ de lignes secondaires en tout. $\mathrm{La}$ " taille des branches mortes » advenait quelquefois dans des zones qui se dépeuplaient progressivement, comme par exemple le réseau à voie étroite de la Sicile, et parfois au contraire dans des zones qui se développaient d'un point de vue économique et démographique mais où le taux de motorisation privée était déjà important, comme par exemple sur la ligne Pise-Tirrenia-Livorno qui fut supprimée en 1961. La séparation entre les systèmes de transport, d'un côté, et la faible et peu clairvoyante planification économique et territoriale, de l'autre, entrainèrent cependant de graves erreurs dans la gestion des infrastructures entre les années 1950 et les années 1970. 
Revue d'histoire des chemins de fer 24-25 (printemps - automne 2001)

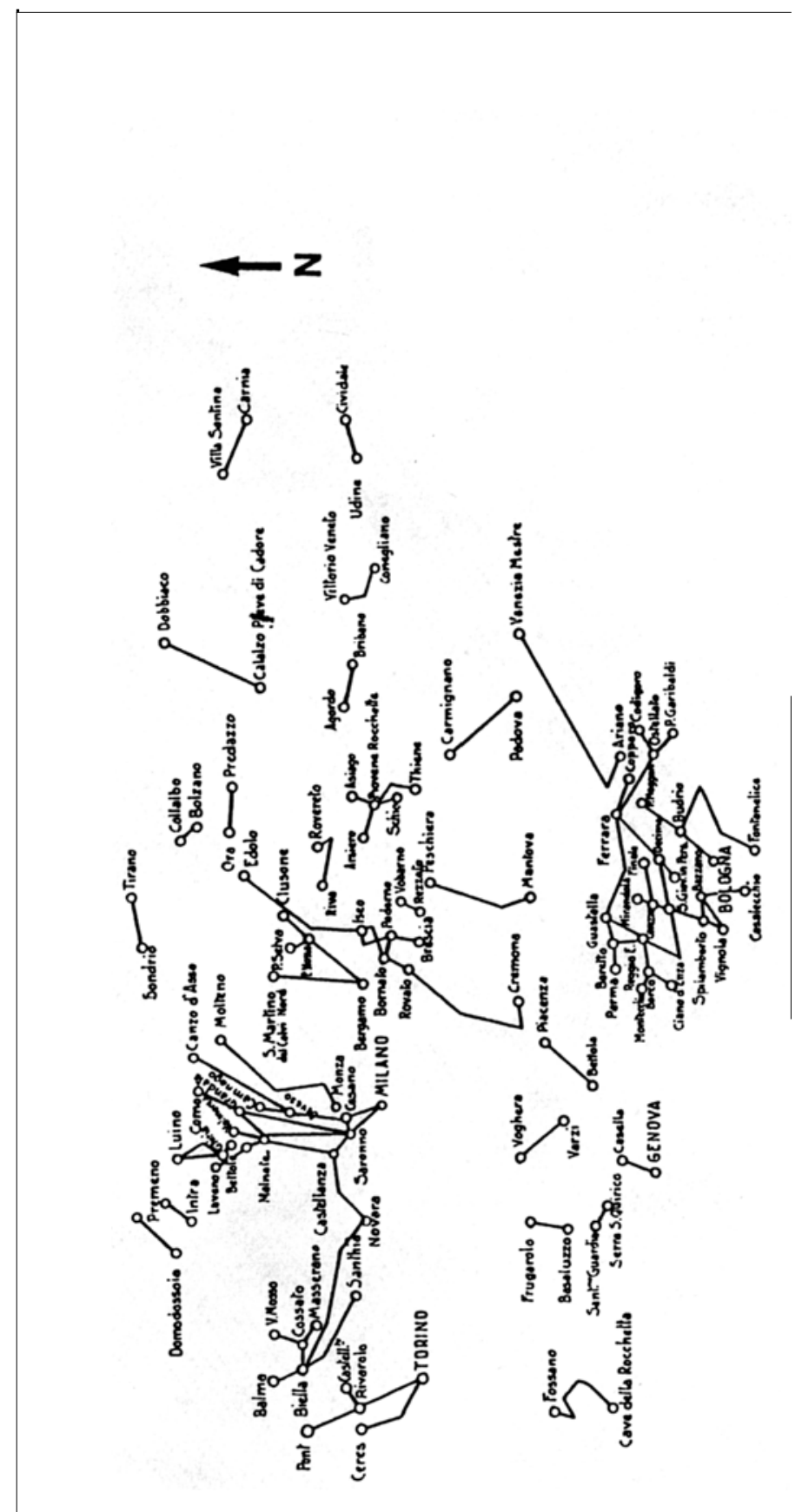




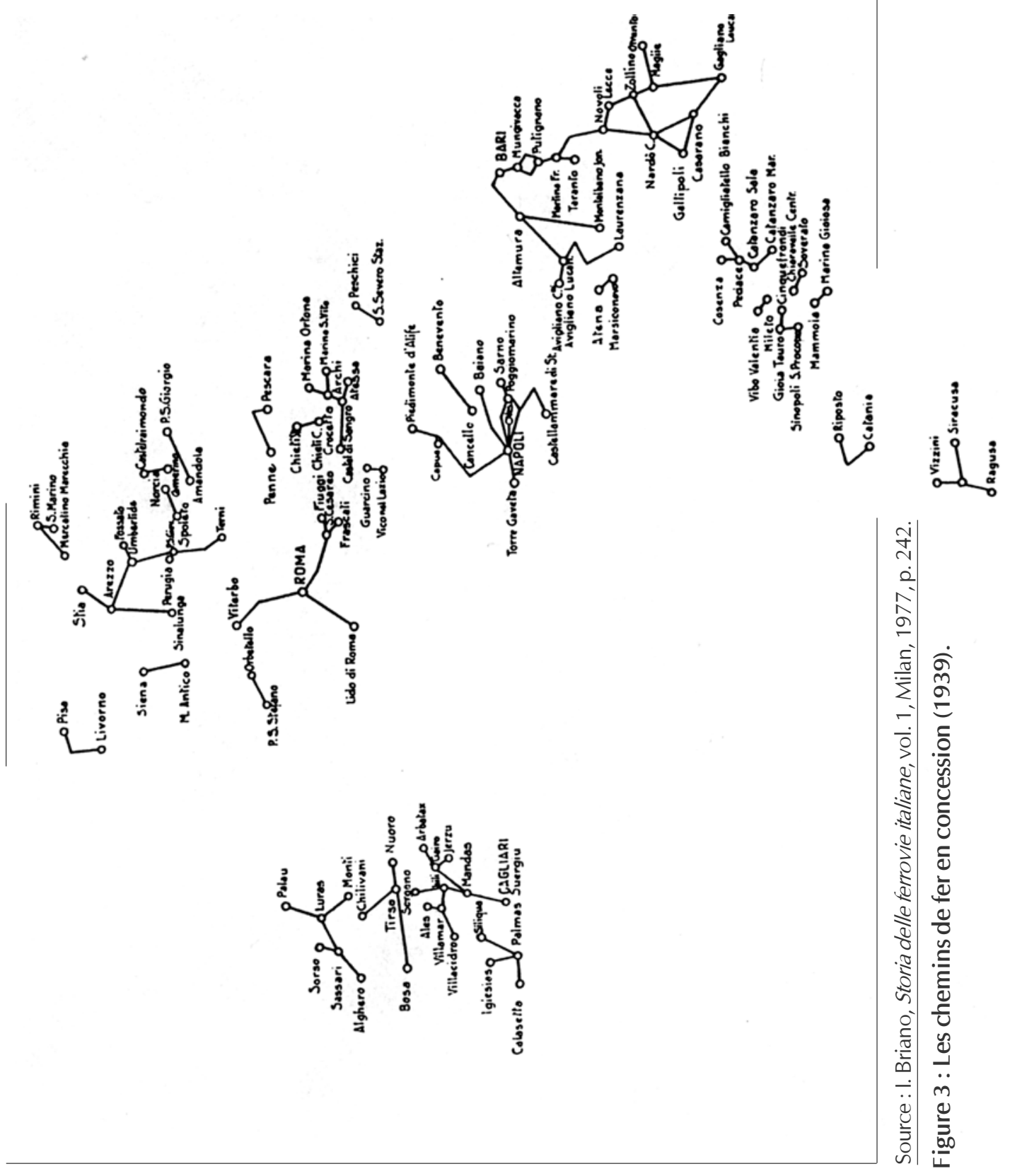


A une échéance plus ou moins lointaine, il est certain que la suppression d'un chemin de fer comportait toujours des conséquences négatives pour un territoire que la diffusion des véhicules automobiles ne réussissait pas à combler complètement. Là où ces effets furent moins importants, on regretta le train pour sa sécurité et sa commodité. Il suffit de citer ici un seul exemple, celui du chemin de fer secondaire pour Cortina d'Ampezzo - la ligne Calalzo-Dobbiaco - qui aurait pu assurer une liaison efficace pour les nombreux visiteurs qui se rendaient dans cette petite ville en hiver et en été, à la suite de l'exceptionnelle augmentation du nombre des déplacements touristiques due à l'élévation générale du niveau de vie et aux changements de la société italienne.

Une dernière réflexion s'impose concernant la relation entre les chemins de fer secondaires et le territoire, étant donné que la présence des rails contribua souvent à provoquer une nouvelle répartition de l'habitat dans les zones de périphérie. Le développement de l'urbanisation autour des gares de chemins de fer provoqua une consequence tout à fait particulière à l'Italie : en effet, étant donné que les nombreux centres médiévaux sont construits sur les collines, ces villages se dédoublèrent et furent renforcés par d'autres villages homonymes et construits dans la plaine ; on ajoutait souvent au nom des villages le terme «scalo » - qui veut dire gare - pour indiquer justement qu'ils s'étaient formés autour d'une gare. Et au fil du temps, ce centre " gare ", grâce aux activités commerciales et manufacturières, était souvent devenu plus important que le centre correspondant situé sur la colline.

Comme je l'ai déjà dit, la notion de «chemin de fer secondaire » ne fut introduite qu'en 1879 et concerna quelques courts tronçons nouvellement construits à ce moment qui reliaient certains centres aux lignes principales. Auparavant, il n'existait aucune réelle différence entre les tronçons principaux et les tronçons secondaires, ni d'un point de vue réglementaire ni d'un point de vue technique. Une différence émergea dans la pratique à la fin du XIXe siècle, quand le réseau national fut terminé, entre les lignes supportant les gros trafics nord-sud ou estouest et les lignes gérant seulement le service local, indépendamment du fait que la gestion en était confiée à des sociétés locales ou à de grandes compagnies concessionnaires (puis aux Chemins de fer de l'État après 1905). C'est à ce moment-là que l'on commença à utiliser un matériel roulant différent, et à négliger les tronçons aux trafics faibles. Mais la véritable séparation s'opéra pendant les vingt années du fascisme, quand les chemins de fer principaux s'électrifièrent quelquefois et que leurs voies doublèrent et commencèrent à être parcourus par des trains rapides, entrainant ainsi le développement économique et démographique des villes les plus importantes situées sur leur itinéraire. 


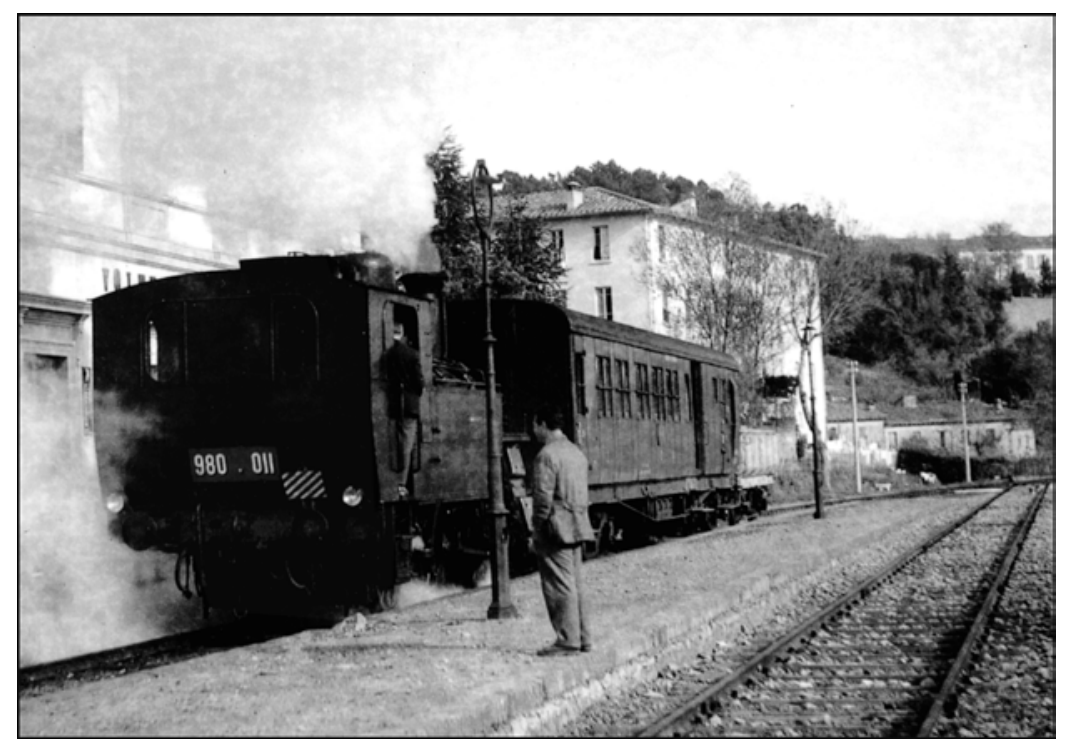

Figure 4 : Train à vapeur (1950) du chemin de fer secondaire à crémaillère Saline di Volterra - Volterra, construit en 1912 et fermé en 1958. Coll. Neri Baldi, cl. d.r.

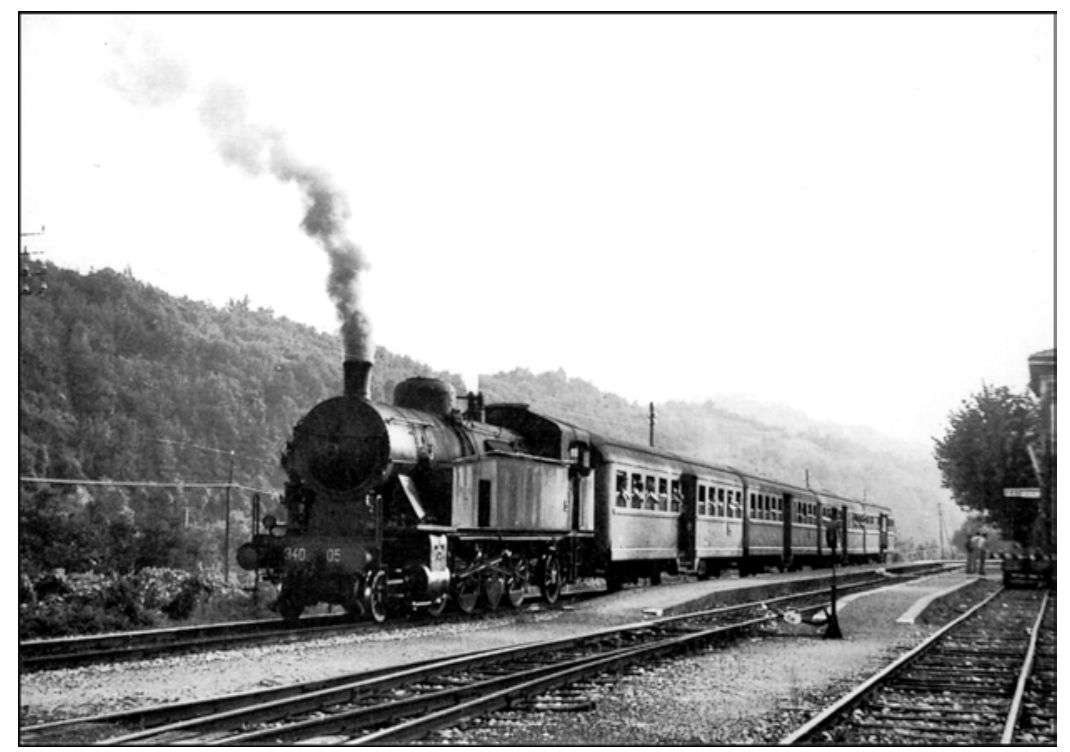

Figure 5 : Chemin de fer Lucca - Aulla (1975), derniers services voyageurs à vapeur.

Coll. Leonardo Carnesecchi, cl. d.r. 
Dans le même temps, les lignes secondaires étaient toujours plus isolées mais avaient malgré tout des effets importants sur le territoire, jusque dans les années 1960 au moins, comme l'augmentation continuelle des villages-gares et l'afflux dans les gares de tous les produits des terres environnantes. Seule la motorisation priva les lignes secondaires de leur importance économique et sociale en les confinant dans un rôle tout à fait marginal pour ce qui concerne le trafic des marchandises et le trafic des voyageurs.

Pourtant, pendant plus de vingt ans, de 1960 à 1985 environ, les chemins de fer secondaires italiens survécurent à la perte de leur rôle. Les lignes survivantes continuaient à fonctionner d'une manière anachronique, avec les mêmes trains, avec les mêmes installations, souvent avec les mêmes cheminots. Les secondaires perdaient marchandises et voyageurs, mais restaient en exploitation sans changements. Ils étaient tout à fait en phase avec l'immobilisme de l'administration italienne du second après-guerre. A cause de l'absence de mise en place d'automatismes, les gares de ces lignes sont restées pendant toute la période 1960-1990 pleines de cheminots, les passages à niveau sont restés gardés et les lignes sont donc en vie davantage pour la présence des travailleurs que pour le trafic existant.

Cette situation a été modifiée brusquement au debut des années 1990, par la réorganisation des Chemins de fer de l'État et des chemins de fer en concession : tout à coup, on s'est aperçu que les lignes secondaires étaient trop coûteuses et qu'elles fonctionnaient mal. Mais au lieu d'essayer de les régénérer, on a supprimé le personnel. Le resultat est sous les yeux de quiconque voyage sur un chemin de fer secondaire italien de nos jours : gares voici quelques années propres et prospères, aujourd'hui abandonnées et en proie aux vandales. Le nombre des trains a été reduit et le transport des marchandises et bagages presque abandonné. Quelques lignes ont été fermées à l'exploitation. En général le nombre des voyageurs est limité à cause de l'inefficacité du service, d'une politique de promotion insuffisante, quelquefois même par la difficulté qu'ils rencontrent à acheter leur billet.

La récente regionalisation du transport ferroviaire est arrivée dans ce contexte de décadence : dorénavant les régions décideront quel service elles financeront sur les chemins de fer de leur territoire. La situation actuelle est caractérisée par une notable disparité d'interventions : quelques regions, mais aussi quelques provinces et communes sont passées à l'action avec efficacité. De toute façon, la mise en valeur des chemins de fer secondaires par le tourisme est presque partout inexistante, alors que dans beaucoup de territoires italiens elle peut répresenter la clef du succès, comme le démontre le cas de la ligne Sienne-Grosseto en Toscane. 


\section{Étude de cas : la ligne Sienne-Grosseto (Toscane)}

Le cas de la voie qui relie Sienne et Grosseto en Toscane illustre parfaitement les vicissitudes des chemins de fer secondaires italiens. Elle avait été réalisée avant que la distinction entre lignes principales et secondaires n'apparaisse, pour relier d'un côté une zone sous-développée à cause du paludisme endémique, la province de Grosseto - appelée couramment Maremme - et de l'autre une ville historique importante, Sienne. Cette voie de communication fut intégrée au réseau par un tronçon construit par la suite, preuve évidente du mouvement de ramification du réseau ferroviaire qui se développa entre la fin du XIX et le début du XX $X^{\mathrm{e}}$ siècles.

La première ligne, projetée en 1860, fut terminée rapidement en mai 1872 pour satisfaire les demandes pressantes des communes qui voulaient qu'un trafic de marchandises soit assurée ${ }^{21}$; ce trafic était beaucoup plus important et son importance plus vivement ressentie que le trafic des voyageurs, resté pour sa part faible pendant longtemps.

Les résultats économiques de cette ligne ne furent jamais particulièrement satisfaisants, au point que le passif s'élevait à environ 60000 lires par an au début du $\mathrm{XX}^{\mathrm{e}}$ siècle $^{22}$. Il existait cependant un important trafic de mercure venu de Monte Amiata qui représentait en 1910 un quart de la production mondiale du cinabre, et un trafic de produits agricoles; on transportait aussi les produits des premières manufactures qui s'installèrent à proximité des gares après l'implantation du chemin de fer. De plus, plusieurs sites ferroviaires devinrent la destination d'un flux de lignite, le combustible que l'Italie utilisait pour ne plus dépendre de l'étranger et pour éviter l'importation du coke ${ }^{23}$.

Pour transporter la lignite, on construisit même un chemin de fer industriel d'environ $20 \mathrm{~km}$ partant d'une gare de cette ligne, Monte Antico, jusqu’à Murlo. Les gares devinrent donc des centres de répartition et de transformation des marchandises. En outre, dans les bâtiments ferroviaires, on ouvrait des buffets et des débits de boisson qui devenaient petit à petit de véritables lieux de sociabilité. Sur la ligne Sienne-Asciano-Grosseto, il y avait de nombreux établissements publics à l'intérieur des gares : comme le territoire environnant n'avait pas de bourgs déjà développés (les villages étaient situés en effet sur les collines), l'agglomération puisait son dynamisme dans la gare de chemin de fer. Dans les dix premières années du XXe siècle, cafés et restaurants s'étaient déjà installés dans la plupart des gares. Le train favorisa aussi le développement des fêtes villageoises pour lesquelles on organisait des convois spéciaux, et le succès du tourisme thermal : en effet, dans les années 1910, on construisit un arrêt sur cette ligne, 
Revue d'histoire des chemins de fer 24-25 (printemps - automne 2001)

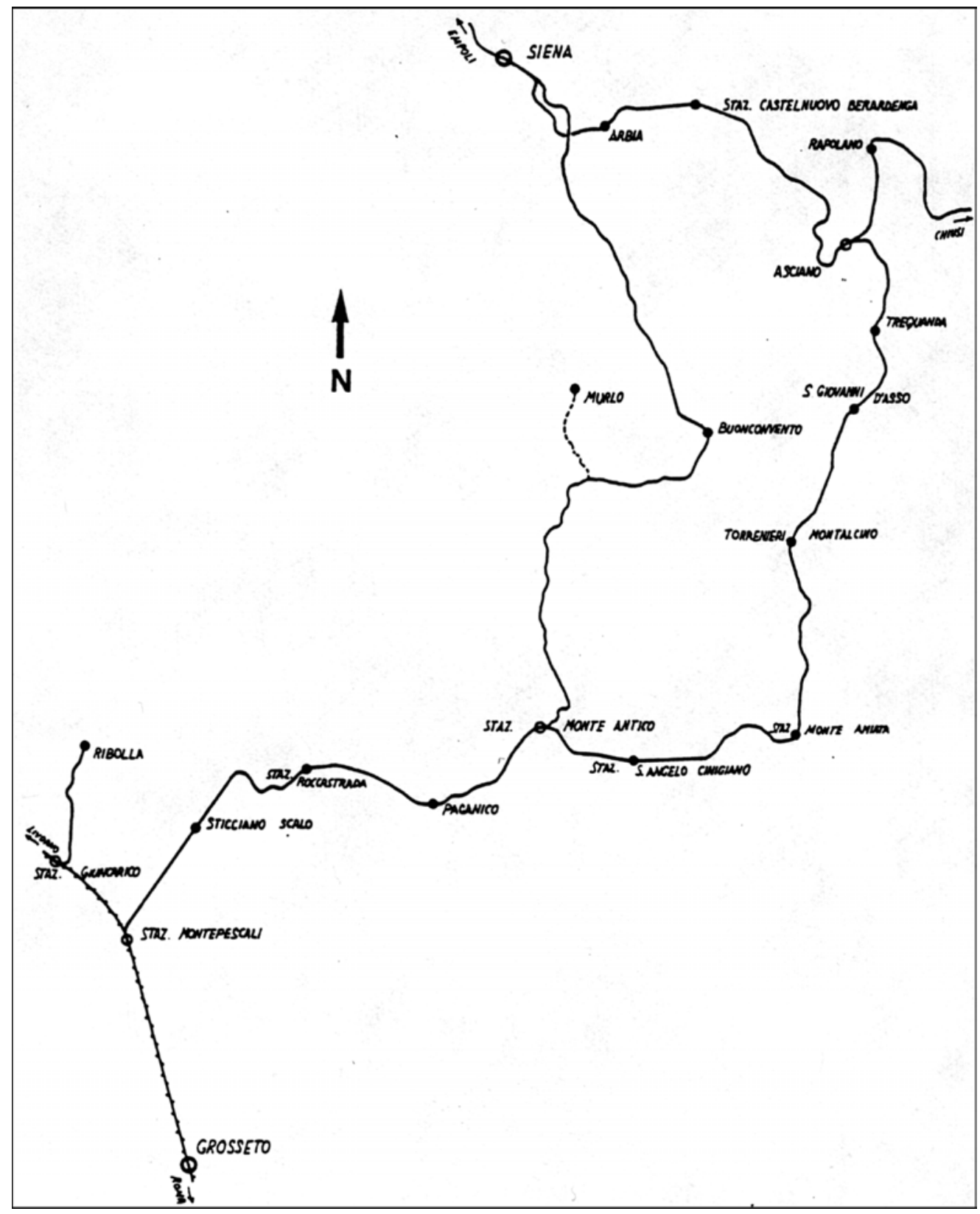

Figure 6 : Les chemins de fer Siena-Grosseto. 


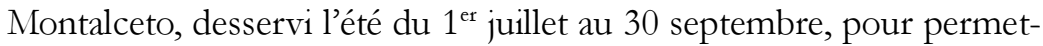
tre aux voyageurs de se rendre à une source d'eau minérale, de s'y baigner, et de rentrer ensuite chez eux le même jour.

Après la Première Guerre mondiale surtout, on commença à construire des bâtiments autour des gares. Il s'agissait de magasins et de remises, de logements pour les cheminots, d'entrepôts de minerais ou de bois et aussi du siège des associations d'agriculteurs. Par la suite, le développement du commerce et des déplacements journaliers des travailleurs attira de nouveaux habitants et entraina ainsi la construction de plusieurs bâtiments à usage d'habitation ${ }^{24}$.

Même dans les cas où le centre urbain avait des origines médiévales anciennes comme Asciano et San Giovanni d'Asso, la présence d'une gare représentait toujours un pôle d'attraction et un motif d'immigration.

Pour le trafic qui existait entre Sienne et la Maremme, la ligne était sans doute suffisante : il s'agissait d'un chemin de fer secondaire, même s'il avait d'abord été géré par les grandes compagnies concessionnaires puis par les Chemins de fer de l'État à partir de 1905. Mais, à la fin du siècle, quand le développement du réseau secondaire commença, certaines communautés locales, exclues de l'itinéraire, exercèrent de fortes pressions pour la réalisation d'une nouvelle voie de communication. Quand le projet fut élaboré, on institua en 1899 une commission technique et juridique qui entama les négociations avec une société étrangère pour la fourniture d'une partie des capitaux.

La demande de concession fut présentée au ministère des Travaux publics par les Communes de Buonconvento et Murlo. Depuis la sortie de Murlo jusqu’à Monte Antico la ligne suivit le tracé du chemin de fer industriel realisé en 1877.

Pour obtenir l'autorisation officielle, la commission énumérait la grande quantité de matières premières et de produits agricoles que le train pourrait desservir et développer en attirant l'industrie.

« La terre est riche en vignes, oliviers et blé. Les bois nombreux et touffus sont une nouvelle source de gains si l'on nous donne des moyens de transport. Les Municipalités ont de nombreuses fêtes et beaucoup de marchés [...] Les mines et les carrières sont à disposition de l'industrie privée. Il y a des mines de lignite à Murlo $[\ldots]$; des mines de manganèse à Montalcino ; $[.$.$] des$ minerais pétrolifères à Buonconvento ; [...] de l'albâtre à Montalcino et à Grosseto; du travertin à Murlo, Montalcino et Grosseto. 


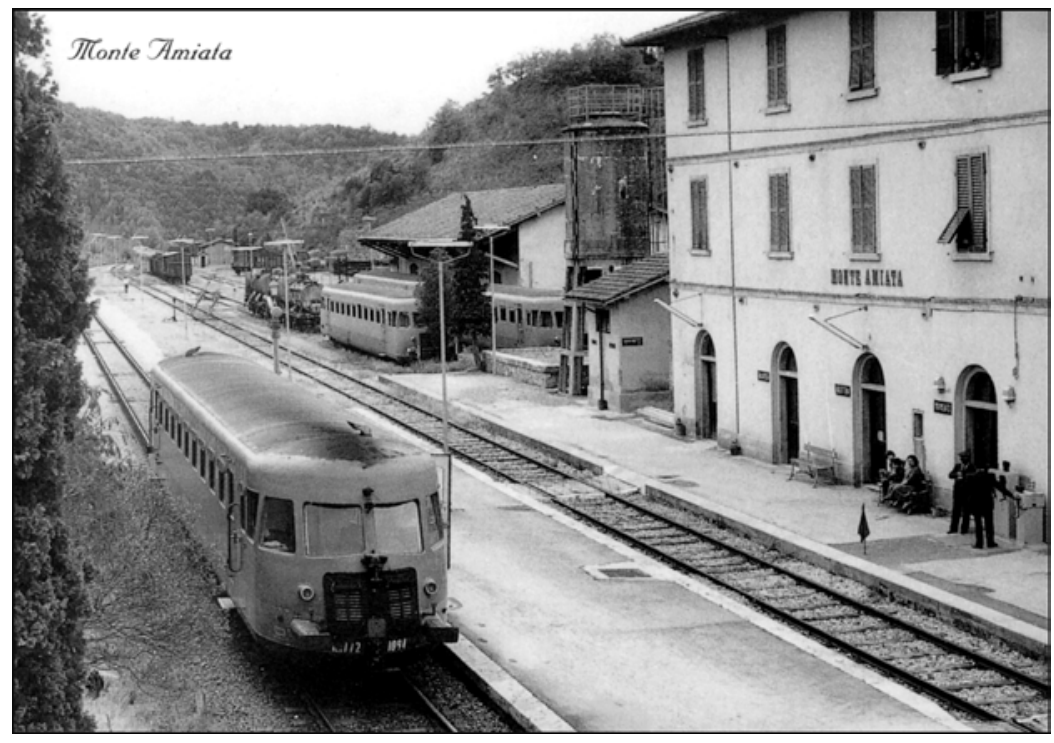

Figure 7 : Chemin de fer Asciano - Monte Antico en 1985. La gare de Monte Amiata avec la gare de marchandises encore en exercice. Coll. Leonardo Carnesecchi, cl. d.r.

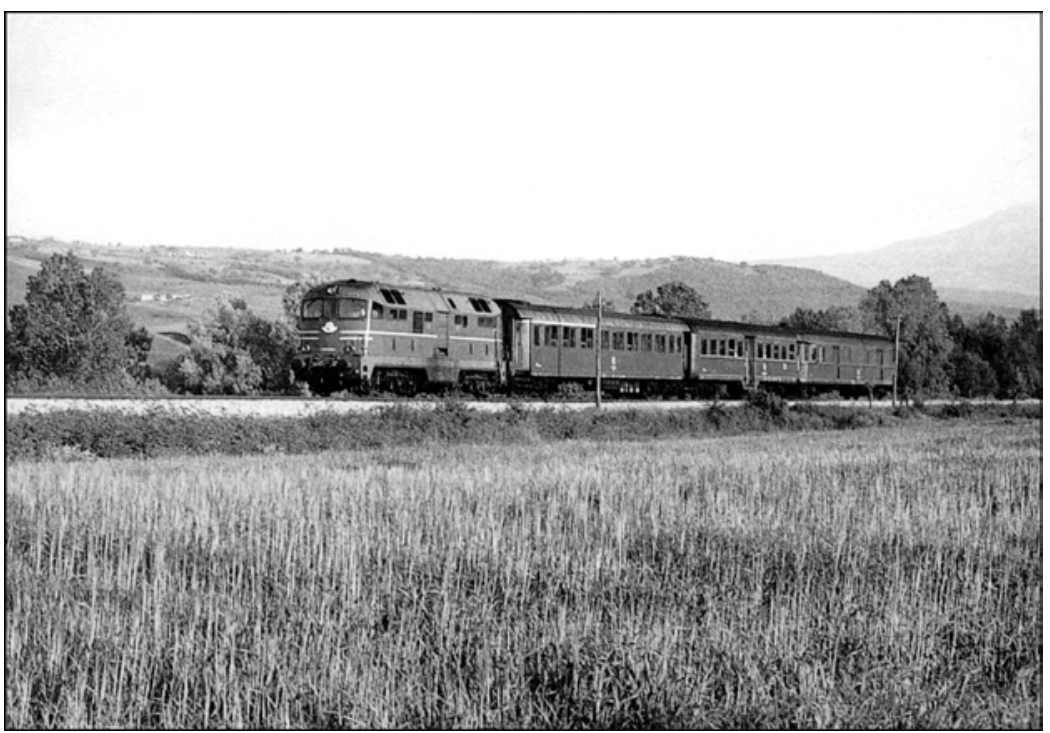

Figure 8 : Train Grosseto - Siena pour voyageurs et bagages. Coll. Leonardo Carnesecchi, cl. d.r. 
L'initiative industrielle ne s'est pas encore adressée, dans nos provinces, à tous les trésors prodigués par la nature. Mais les entreprises qui seront fondées après la construction du chemin de fer deviendront un facteur de prospérité et de progrès économique ${ }^{25}$.»

L'énergie de la commission et la volonté tenace des communes intéressées, associées aux pressions politiques des députés locaux, furent déterminantes dans la lente réalisation du projet. En effet, pendant les mêmes années, les autres chemins de fer proposés pour les provinces de Sienne et Grosseto connurent un échec, malgré leur plus grande utilité et la conformité des projets aux critères fixés par la réglementation pour leur financement. Il s'agissait de projets typiques de réseaux secondaires déjà dépassés à cause de la diffusion concomitante des véhicules automobiles et de l'ouverture des premières lignes de cars que l'on commença à mettre en service en janvier 1913, précisément sur un itinéraire (Sienne-Massa Marittima) pour lequel la construction d'une ligne de chemin de fer avait été prévue. Par la suite une route fut inaugurée, avec cérémonie officielle, également sur le parcours SienneGrosseto, en mars $1920^{26}$.

Mais voyons brièvement l'histoire de la construction de la seconde ligne de communication entre Sienne et la Maremme. Le projet fut présenté au Conseil supérieur des travaux publics qui l'accepta sous réserves, en demandant quelques modifications. Un nouveau rapport technique fut alors élaboré et le conseil approuva définitivement le projet de la ligne Sienne-Buonconvento-Monte Antico le 15 mai 1905 ; le ministère du Trésor lui accorda en 1907 une contribution annuelle de 5 000 lires par kilomètre pour une durée de 70 ans. Un public nombreux assista à la séance du Conseil de la Province de Sienne au cours de laquelle on décida qu'un subside serait affecté au chemin de fer en construction, ce qui prouve l'importance qu'elle revêtait pour les populations. Ce chemin de fer fut donc réalisé grâce à des capitaux garantis par l'État et par les organismes locaux, tandis que sa construction et sa gestion furent confiées à une société privée, conformément au modèle le plus répandu des chemins de fer secondaires de la fin du XIX et du début du XX $\mathrm{XX}^{\mathrm{e}}$ siècle .

Une convention pour la réalisation de la ligne fut passée avec la Société française de chemin de fer en Toscane en décembre 1910. Les travaux commencèrent en 1912 mais se poursuivirent à un rythme lent, car s'ajoutèrent les modifications qu'une orographie difficile imposèrent au tronçon proche de Sienne. L'emplacement de la gare de Buonconvento posa d'autres problèmes : pour la rapprocher le plus 
possible de l'agglomération, comme demandait le maire, on apporta une modification au projet ${ }^{27}$. Pendant la Grande Guerre, les travaux furent suspendus et, en 1919, on n'avait achevé que la voie et les bâtiments. En février 1922, la concession pour la construction de la ligne passa à la Société italienne des entreprises publiques et des chemins de fer (SIF) qui continua les travaux assez rapidement et les termina à la fin de 1926, avec malgré tout quatre ans de retard sur le délai prévu par le décret de concession. Quand les essais indispensables furent terminés, le chemin de fer, dont la gestion fut confiée à la société qui l'avait construit, fut inauguré officiellement le 30 mai 1927. A partir de cette date, les communications entre Sienne et la Maremme, axe lui-même secondaire et susceptible d'un faible trafic, furent assurées par deux lignes : Sienne-Asciano-Monte Antico-Grosseto, gérée par les Chemins de fer de l'État, et Sienne-Buonconvento-Monte Antico, gérée par la SIF.

La ligne de Buonconvento fut par la suite rachetée par l'État et intégrée dans le réseau national à partir de mars 1956, suite à la résiliation de la concession avec la SIF qui rencontrait d'importants problèmes économiques. Dix ans plus tard, on suspendit le service voyageurs du tronçon Sienne-Buonconvento car il figurait sur la liste des «branches mortes » et on remplaça le train par des cars. Le tronçon BuonconventoMonte Antico restait en service à cause de l'absence complète de routes parallèles qui rendait irremplaçable le rôle du train pour la population. La ligne Sienne-Buonconvento demeura fermée pendant 24 ans jusqu'à sa réouverture en 1980.

L'élimination de l'une des deux voies de communication entre Sienne et la Maremme ne résolut pas cependant les problèmes de l'autre.

Avec les nouvelles technologies et l'essor de la motorisation individuelle, la ligne Asciano-Grosseto était devenue trop longue pour assurer la liaison entre les deux chefs-lieu de province $(129 \mathrm{~km}$ de voie ferrée contre $80 \mathrm{~km}$ de route) ; par ailleurs, elle traversait un territoire comprenant peu d'agglomérations et son tracé avait été conçu spécialement pour le chemin de fer, car il n'existait avant lui aucune autre voie routière sur le même tracé. De plus, un tronçon de la ligne souffrait de la concurrence des services d'autocars, même s'il s'agissait d'une portion limitée du parcours.

L'analyse du trafic des usagers et de celui des marchandises sur une longue période montre que cette infrastructure a toujours été faiblement utilisée quand on la compare à la moyenne et met en évidence une chute prononcée du trafic à partir de 1970. Si l'on prend comme base l'année 1910, année où les effets de la réorganisation des chemins 
de fer commençaient à se manifester suite à la nationalisation, et si l'on compare ces chiffres avec ceux de l'année 1928 (avant la crise de 1929) et avec d'autres du second après-guerre ${ }^{28}$, on peut noter une hausse progressive du trafic dans les trente premières années du XXe siècle.

Dans l'ensemble, entre l'année 1910 et l'année 1928, le chemin de fer développa tant le trafic voyageurs que celui des marchandises : la moyenne des voyageurs annuels par $\mathrm{km}$ de ligne augmenta en effet de 531 à 670. Dans le second après-guerre, les déplacements augmentèrent encore avec l'importante croissance économique de l'Italie et l'on passa ainsi, en 1967, à 1926 usagers par km ; mais, dans les années qui suivirent, leur nombre baissa régulièrement pour atteindre 1796 voyageurs par km en 1971, 1318 en 1981 et, enfin, 1085 en 1992.

Les données du second après-guerre relatives aux marchandises sont fluctuantes jusque dans les années 1980 : si l'on prend comme référence les tonnes $/ \mathrm{km}^{29}$, on peut observer une chute prononcée : de 2995000 tonnes/ $\mathrm{km}$ en 1967 à 1637000 en 1971. Il y eut ensuite une remontée, atteignant les 1801000 tonnes en 1981, mais elle a été suivie d'une chute définitive, jusqu'à 205000 tonnes en 1992, quand le trafic des marchandises a pratiquement disparu à cause de la fermeture progressive des gares qui, quelques années auparavant, étaient encore autorisées à expédier des wagons de marchandises complets et pouvaient de ce fait réaliser un certain bénéfice.

L'agonie de la ligne, sur le tronçon Asciano-Monte Antico, commença à la fin des années 1980, quand on supprima non seulement le transport des marchandises mais aussi, l'une après l'autre, les six allerretour de trains voyageurs qui circulaient tous les jours. Le tronçon suivant Monte Antico fut intégré à la ligne de communication à moyenne distance - Florence-Sienne-Grosseto - mise en service en mai 1980, au moment de la réouverture de la ligne Sienne-Buonconvento, ligne qui fut alors renforcée et dotée d'un équipement moderne de régulation.

Au cours des premiers mois de l'année 1994, le bruit courut que l'on allait supprimer le service des voyageurs, et les habitants de San Giovanni d'Asso se mobilisèrent car cette petite ville était la seule municipalité vraiment intéressée à la ligne car elle n'était reliée à aucune autre voie valable. Mais il s'agissait de la plus petite commune de la Province, comptant moins de 1000 habitants. Des pressions politiques bloquèrent la taille de cette nouvelle "branche morte " pour l'été qui allait suivre et on laissa circuler seulement deux aller-retour les jours ouvrables, tandis que les jours fériés la ligne était complètement déserte. Mais la fermeture de la ligne n'avait été reportée que de quelques semaines : en effet, dans l'horaire de l'hiver qui suivit, tous les trains ordinaires étaient définitivement supprimés. 
Deux ans plus tard, la valorisation de cette ligne par le tourisme commença avec le projet « Train Nature », financé par l'administration de la Province de Sienne avec la collaboration d'une association de bénévoles. Les jours fériés, au printemps et en automne, on peut voir circuler des trains à vapeur et des autorails d'époque sur l'ancien tracé de 1872 ; ces trains assument un rôle économique et social nouveau et se mettent au service d'un territoire - les Crêtes siennoises et la Vallée de l'Orcia - qui est, entre autre, la patrie du célèbre vin « Brunello » et l'une des plus importantes destinations du tourisme de «qualité » en Italie $^{30}$.

\section{Notes}

1- Pourtant les débats avaient commencé depuis longtemps, suivant l'exemple de la loi française du 1865. Voir A. Barchetta, Sulla costruz̨ione delle ferrovie secondarie. Considerazioni economiche, tecniche e finanziarie, Turin, 1866 ; F. Biglia, Le ferrovie economiche d'Europa. Relazione al signor Ministro dei Lavori Pubblici, Florence, 1867. La première loi sur les chemins de fer secondaires fut présentée au Parlement en février 1866, mais ne fut pas votée.

2- P. Valussi, «Le piccole città nel nuovo ordinamento d'Italia », Nuova Antologia, vol. VIII (juillet 1868), p. 541.

3- Loi 29 juillet $1879 n^{\circ} 5002$.

4- M. Merger, «Le ferrovie dell'Italia settentrionale », in La rivoluzione dei trasporti in Italia nel XIX secolo, sous la dir. de G. Sabatini, L'Aquila, 1996, p. 80.

5- A. Crispo, Le ferrovie italiane. Storia politica ed economica, Milan, 1940, pp. 197-201 et p. 122.

6- En 1865 la construction de voies ferrées ainsi que leur gestion furent données en concession à trois sociétés à capital privé : les Chemins de fer de l'Italie du Nord, les Chemins de fer romains et les Chemins de fer de l'Italie du Sud. En 1885, par la « loi sur les conventions » la péninsule fut confiée à la Société des Chemins de fer de la Méditerranée pour sa partie ouest (Réseau méditerranéen) et à la Société des Chemins de fer du Sud pour sa partie est (Réseau adriatique); la Société des Chemins de fer de la Sicile, enfin, recevait en concession les lignes de l'île.

7- Ministero dei Lavori Pubblici, Relazione sull'esercizio delle strade ferrate per l'anno 1905, Rome, 1909, p. X.

8- Pour les premiers tramways italiens, voir A. Viappiani, La costruzione e l'esercizio delle tramive, Turin, 1893.

9- «La costruzione e l'esercizio delle ferrovie italiane nel $1878 »$, Giornale del Genio Civile (1880, partie non-officielle), pp. 191-199. 
10- P. Hertner, « Municipalizzazione e capitale straniero nell'età giolittiana », in $L a$ municipalizzazione nell'area padana. Storia ed esperienze a confronto, Milan, Angeli, 1988, p. 59.

11- «Le tramvie a vapore in Italia al $1^{\text {er }}$ ottobre $1888 »$, Monitore delle Strade Ferrate (1888), p. 746 et pp. 778-780.

12- W. Guadagno, Ferrovie ed economia nell'Ottocento post-unitario, Rome, CAFI, 1995 , p. 265.

13- A. Giuntini, «Nascita, sviluppo e tracollo della rete infrastrutturale », in Storia d'Italia. Annali. Vol. XV, L'industria, Turin, Einaudi, 1999, p. 601.

14- G.E. Baddeley, The continental Steam Tram, Londres, 1980, pp. 178-180.

15- W. Guadagno, Ferrovie ed economia nell'Ottocento post-unitario, op. cit., p. 264.

16- Loi du 27 décembre 1896 n 561.

17- Voir, pour une histoire sociale de ce chemin de fer, E. Caglieri, Il trenino per Vallombrosa. Storia di una ferrovia e di un ferroviere a scartamento ridotto, Florence, 1977.

18- P. Lanino, «L'industria privata nella ferrovia », Ingegneria Ferroviaria (janvier 1918), p. 82.

19-Voir G. Fumi, "Vie di comunicazione e trasporti », in Guida all'Italia contemporanea. Vol. I, Risorse e strutture economiche, Milan, Garzanti, 1998, p. 103.

20- ISTAT, Annuario statistico italiano, 1930 et 1939.

21- S. Maggi, La ferrovia per la Maremma. Con la nuova edizione del Viaggio in strada ferrata da Asciano a Grosseto di G. Losi, Sienne, Nuova Immagine Editrice, 1996, p. 29.

22- «A proposito di ferrovie », La Martinella Siena Nuova, 10 août 1907.

23- A. Giuntini, «La grande illusione. La scoperta del carbon fossile nella Toscana della prima metà dell'Ottocento », Ricerche Storiche, n 1 (1989), pp. 3-43.

24- M. Sorelli, «L'evoluzione degli insediamenti maremmani tra la metà del ‘700 e gli anni ‘50 del ' $900 »$, in La Maremma grossetana tra il '700 e il '900. Trasformazioni economiche e mutamenti sociali, Città di Castello, 1989, p. 162.

25- C. Camera, Sul progetto di ferrovia economica Siena-Buonconvento-Monte Antico e Buonconvento-Torrenieri, Sienne, 1900, p. 15.

26- «L'inaugurazione del servizio automobilistico Siena-Grossetto », La Vedetta Senese, $1^{\text {er }}$ et 2 avril 1920.

27- Ferrovia Siena-Buonconvento-Montantico, Proposta di variante alla ubicazione della staz̧ione di Buonconvento progettata dalla Società concessionaria. Relazione, Sienne, 1911.

28- Les chiffres sont pris dans les publications suivantes : Ferrovie dello Stato, Statistica dell'esercizio anno 1910, parte I, Statistica generale, e parte II, Statistica del 
traffico, Rome, 1912 ; Ferrovie dello Stato, Traffico viaggiatori e merci diviso per stazione anno 1928, Rome, 1929; Ferrovie dello Stato, Principali dati caratteristici del traffico per linea statistica, 1967 ; Ferrovie dello Stato, Principali dati caratteristici del traffico per linea statistica, 1971 ; Ferrovie dello Stato, Principali dati del traffico per linea statistica, 1981 ; Ferrovie dello Stato, Principali dati del traffico per linea statistica, 1992.

29- Calcul obtenu à partir des tonnes en circulation sur une ligne, nombre multiplié par la moyenne kilométrique du trajet.

30- Voir S. Maggi, «Conservation et mise en valeur du patrimoine ferroviaire par le tourisme : l'exemple du Train Nature, train touristique Asciano-Monte Antico (Toscane) », in «Le Patrimoine ferroviaire : enjeux, bilans et perspectives », Revue d'histoire des chemins de fer, $\mathrm{n}^{\circ} 20-21$ (printemps-automne 1999), pp. 276-292 ; et le guide Viaggio sul Treno Natura. Guida storico-paesaggistica con 8 itinerari escursionistici, sous la dir. de S. Maggi, Sienne, Nuova Immagine Editrice, 1998. 